\title{
Autophagy and apoptosis induction by sesamin in MOLT-4 and NB4 leukemia cells
}

\author{
KAMOLCHANOK DEESRISAK ${ }^{1}$, CHAWALIT CHATUPHEERAPHAT ${ }^{1}$, \\ SITTIRUK ROYTRAKUL ${ }^{2}$, USANARAT ANURATHAPAN ${ }^{3}$ and DALINA TANYONG ${ }^{1}$
}

\author{
${ }^{1}$ Department of Clinical Microscopy, Faculty of Medical Technology, Mahidol University, Nakhon Pathom 73170; \\ ${ }^{2}$ Proteomics Research Laboratory, National Center for Genetic Engineering and Biotechnology, \\ Thailand Science Park, Pathum Thani 12120; ${ }^{3}$ Department of Pediatrics, Faculty of Medicine Ramathibodi Hospital, \\ Mahidol University, Bangkok 10400, Thailand
}

Received January 20, 2020; Accepted October 16, 2020

DOI: $10.3892 / 01.2020 .12293$

\begin{abstract}
Sesamin, the major furofuran lignan found in the seeds of Sesamum indicum L., has been investigated for its various medicinal properties. In the present study, the anti-leukemic effects of sesamin and its underlying mechanisms were investigated in MOLT-4 and NB4 acute leukemic cells. Leukemic cells were treated with various concentrations of sesamin. Cell viability was determined using an MTT assay. Flow cytometry using Annexin V-FITC/PI staining and anti-LC3/FITC antibodies was applied to detect the level of apoptosis and autophagy, respectively. Reverse transcription-quantitative PCR was performed to examine the alterations in the mRNA expression of apoptotic and autophagic genes. In addition, bioinformatics tools were used to predict the possible interactions between sesamin and its targets. The results revealed that sesamin inhibited MOLT-4 and NB4 cell proliferation in a dose-dependent manner. In addition, sesamin induced both apoptosis and autophagy. In sesamin-treated cells, the gene expression levels of caspase 3 and unc-51 like autophagy activating kinase 1 (ULK1) were upregulated, while those of mTOR were downregulated compared with in the control. Notably, the protein-chemical interaction network indicated that caspase 3 , mTOR and ULK1 were the essential factors involved in the effects of sesamin treatment, as with anticancer agents, such as rapamycin, AZD8055, Torin1 and 2. Overall, the findings of the present study suggested that sesamin inhibited MOLT- 4 and NB4 cell proliferation, and induced apoptosis and autophagy through the regulation of caspase 3 and mTOR/ULK1 signaling, respectively.
\end{abstract}

Correspondence to: Dr Dalina Tanyong, Department of Clinical Microscopy, Faculty of Medical Technology, Mahidol University, 999 Phuthamonthon Sai 4 Road, Salaya, Nakhon Pathom 73170, Thailand

E-mail: dalina.itc@mahidol.ac.th

Key words: sesamin, leukemia, apoptosis, autophagy, protein-chemical interaction

\section{Introduction}

Leukemia is a hematological malignancy characterized by an increase of abnormal leukocytes in the blood and bone marrow (1). In 2018, a total of 437,000 new cases and 309,000 deaths of leukemia were estimated worldwide (2). Chemotherapy is the main treatment for leukemia for decreasing the excessive number of leukocytes, but the efficacy is limited by its side effects, such as nausea, hair loss and changes in appetite (3). Therefore, complementary and alternative medicine using natural compounds has been used to enhance the efficacy of leukemia treatment (4). Sesamin is the major oil-soluble furofuran lignan abundantly found in sesame seeds and related products (5). Several studies have reported the medicinal properties of sesamin, including antioxidant (6), anti-inflammatory (7), anti-hypertensive (8) and anti-hypercholesterolemic activities (9). The anticancer activity of sesamin has been revealed in a number of types of cancer, such as lung cancer, breast cancer and leukemia (10). Sesamin is able to inhibit the proliferation and induce apoptosis of lung cancer cells via cyclooxygenase (COX)-2 (11). Additionally, it advocates cell cycle arrest by increasing the levels of p53 and checkpoint kinase 1 phosphorylation, and inducing apoptosis via Bax and caspase 3 in MCF-7 cells (12). Moreover, sesamin has been reported to potentiate autophagy; it triggers autophagy through the regulation of EPH receptor (Eph)A1 and EphB2 in colon cancer cells (13). Additionally, it activates autophagic cell death in the HeLa cervical cancer cell line (14). However, evidence of the anti-leukemic effects of sesamin is limited.

Sesamin possesses anti-leukemic activities by decreasing the proliferation and inducing apoptosis of HL-60 cells via the mitochondrial and endoplasmic reticulum stress pathways (15). It promotes TNF-induced apoptosis by suppressing the expression of survival genes (such as Bcl-2 and survivin), cell proliferative genes (such as cyclin D1 and COX-2) and genes involved in invasion and angiogenesis, including intracellular adhesion molecule 1 and matrix metalloproteinase 9, in KBM-5 cells (16).

Thus, the present study investigated the effects of sesamin on MOLT-4 and NB4 leukemic cells in vitro and examined 
the underlying mechanisms associated with apoptosis and autophagy.

\section{Materials and methods}

Leukemic cell culture and peripheral blood mononuclear cell (PBMC) isolation. The human T-lymphocytic leukemic MOLT-4 cell line and human acute promyelocytic leukemic NB4 cell line were purchased from CLS Cell Lines Service $\mathrm{GmbH}$. The cells were cultured in RPMI-1640 medium supplemented with $10 \% \mathrm{FBS}$ and $1 \%$ penicillin-streptomycin (all from Gibco; Thermo Fisher Scientific, Inc.) in a humidified incubator at $37^{\circ} \mathrm{C}$ with $5 \% \mathrm{CO}_{2}$. The isolation of PBMCs from healthy adult volunteers ( 1 female and 2 males; mean age, 27 years; age range, 26-27 years) was performed using Lymphoprep ${ }^{\mathrm{TM}}$ (Axis-Shield PoC AS) according to the manufacturer's protocol. Briefly, $10 \mathrm{ml}$ of blood was collected from volunteers in August 2019 and diluted with an equal volume of PBS. The diluted blood was gently layered onto the Lymphoprep solution. The separation was done by centrifugation at $800 \mathrm{x} \mathrm{g}$ for $30 \mathrm{~min}$ at $25^{\circ} \mathrm{C}$. The PBMC layer consisting of lymphocytes and monocytes was collected and washed twice with medium before being used for the cell viability assay. All healthy adult volunteers provided written informed consent for donating peripheral blood to the present study, which was in accordance with the guidelines of the Committee for Research of the Faculty of Medicine Ramathibodi Hospital, Mahidol University (Bangkok, Thailand), based on the Declaration of Helsinki.

Determination of cell viability by MTT assay. The effects of sesamin on cell viability were determined using an MTT assay. Sesamin (Sigma-Aldrich; Merck KGaA) was dissolved in DMSO at a stock concentration of $50 \mathrm{mg} / \mathrm{ml}$ and small aliquots were stored at $-20^{\circ} \mathrm{C}$. Immediately before use, individual aliquots were thawed and diluted in cell culture medium to the required concentrations. The leukemic cells and PBMCs were treated with various concentrations of sesamin $(0,100,300$ and $500 \mu \mathrm{g} / \mathrm{ml}$ ). Following 24 and $48 \mathrm{~h}$ of incubation at $37^{\circ} \mathrm{C}$, $5 \mathrm{mg} / \mathrm{ml}$ MTT (Thermo Fisher Scientific, Inc.) was added, followed by incubation for $4 \mathrm{~h}$ at $37^{\circ} \mathrm{C}$. The formazan crystals were dissolved with solubilizing solution (10\% SDS in $0.01 \mathrm{~N}$ $\mathrm{HCl})$ and the optical density was measured at a wavelength of $570 \mathrm{~nm}$ using a spectrophotometer. The $\mathrm{IC}_{50}$ was calculated from linear regression for further experiments.

Analysis of apoptotic cells using flow cytometry. The leukemic cells were treated with sesamin at the $\mathrm{IC}_{50}$ concentration (400 $\mu \mathrm{g} / \mathrm{ml}$ for MOLT-4 and $600 \mu \mathrm{g} / \mathrm{ml}$ for NB4). Following $48 \mathrm{~h}$ of incubation at $37^{\circ} \mathrm{C}$, the cells were washed twice with cold PBS and resuspended in $1 \mathrm{X}$ binding buffer. Apoptosis was examined using the FITC Annexin V Apoptosis Detection kit (BD Biosciences) by staining the cells with $5 \mu \mathrm{l}$ Annexin V and $5 \mu \mathrm{l}$ PI for $15 \mathrm{~min}$ at room temperature in the dark. The apoptotic cells were detected using a FACS Canto II flow cytometer (BD Biosciences) within $1 \mathrm{~h}$ and analyzed using the BD FACSDiva software version 6.1.3 (BD Biosciences).

Analysis of autophagy induction using flow cytometry. The leukemic cells were treated with sesamin at the $\mathrm{IC}_{50}$ concentration $(400 \mu \mathrm{g} / \mathrm{ml}$ for MOLT-4 and $600 \mu \mathrm{g} / \mathrm{ml}$ for NB4) at $37^{\circ} \mathrm{C}$ for $48 \mathrm{~h}$. The LC3-II level was determined using the FlowCellect $^{\mathrm{TM}}$ Autophagy LC3 Antibody-based Assay kit (Merck KGaA) following the manufacturer's protocol. At $30 \mathrm{~min}$ before the end of the time point, $10 \mu \mathrm{l}$ diluted reagent A were added, followed by incubation at $37^{\circ} \mathrm{C}$ for $30 \mathrm{~min}$. The medium was aspirated and $100 \mu \mathrm{l}$ reagent $\mathrm{B}$ were added, followed by immediate spinning. The cells were resuspended in $1 \mathrm{X}$ assay buffer and stained with anti-LC3/FITC antibody for $30 \mathrm{~min}$ at room temperature in the dark, followed by flow cytometric detection using a FACS Canto II flow cytometer (BD Biosciences) within $1 \mathrm{~h}$. The level of LC3-II was calculated from the mean fluorescence intensity analyzed using the BD FACSDiva software version 6.1.3 (BD Biosciences).

Reverse transcription-quantitative PCR (RT-qPCR). Leukemic cells were treated with sesamin at the $\mathrm{IC}_{50}$ concentration $(400 \mu \mathrm{g} / \mathrm{ml}$ for MOLT-4 and $600 \mu \mathrm{g} / \mathrm{ml}$ for NB4) at $37^{\circ} \mathrm{C}$ for $48 \mathrm{~h}$. Total RNA was extracted using GENEzol ${ }^{\mathrm{TM}}$ reagent (Geneaid Biotech Ltd.) and converted to $2 \mu \mathrm{g}$ of cDNA using the RevertAid First Strand cDNA Synthesis kit (Thermo Fisher Scientific, Inc.) according to the manufacturer's protocol. qPCR was performed using the Bio-Rad CFX96 touch ${ }^{\mathrm{TM}}$ real-time PCR detection system (Bio-Rad Laboratories, Inc.) with $2 \mu 1$ template, $0.5 \mu 1$ of each primer, $10 \mu 1$ Luna $^{\circledR}$ Universal qPCR Master Mix (New England Biolabs, Inc.) and nuclease-free water to reach a final volume of $20 \mu \mathrm{l}$. The thermocycling conditions were as follows: Initial denaturation at $95^{\circ} \mathrm{C}$ for $15 \mathrm{sec}$, followed by 40 cycles of denaturation at $95^{\circ} \mathrm{C}$ for $15 \mathrm{sec}$ and extension at $60^{\circ} \mathrm{C}$ for $30 \mathrm{sec}$. The following primers were used for detection: Caspase 3 (CASP3) forward, 5'-TTCAGAGGGGATCGTTGTAGAAGTC-3' and reverse, 5'-CAAGCTTGTCGGCATACTGTTTCAG-3'; mTOR forward, 5'-CGCTGTCATCCCTTTATCG-3' and reverse, 5'-ATGCTCAAACACCTCCACC-3'; unc-51 like autophagy activating kinase 1 (ULK1) forward, 5-'GGCAAG TTCGAGTTCTCCCG-3' and reverse, 5'-CGACCTCCAAAT CGTGCTTCT-3'; and GAPDH forward, 5'-GCACCGTCA AGGCTGAGAA-3' and reverse, 5'-AGGTCCACCACTGAC ACGTTG-3'. GAPDH was used as an endogenous control for normalization. The mRNA expression was calculated from the mean $C_{q}$ value using the $2^{-\Delta \Delta C q}$ method (17).

Prediction of protein-chemical interactions using bioinformatics tools. The interaction network between sesamin and possible targets was predicted using the Search Tool for Interactions of Chemicals (STITCH) version 5.0, which is a search tool used for predicting the interactions of chemicals and proteins that integrates information and databases, including metabolic pathways, crystal structures, binding experiments and drug-target associations to predict biological events (18). The parameters were determined as follows: List of names, sesamin, caspase3, mTOR and ULK1; species, Homo sapiens; confidence score, medium (0.400).

Statistical analysis. The experiments were performed in triplicate. All data are presented as the mean \pm SEM. For comparisons between 2 groups, unpaired Student's t-test was used. For comparisons between $>2$ groups, data were analyzed using a one-way ANOVA followed by Dunnett's multiple comparisons test using GraphPad Prism 6 
<smiles>C1CCOCC1</smiles>
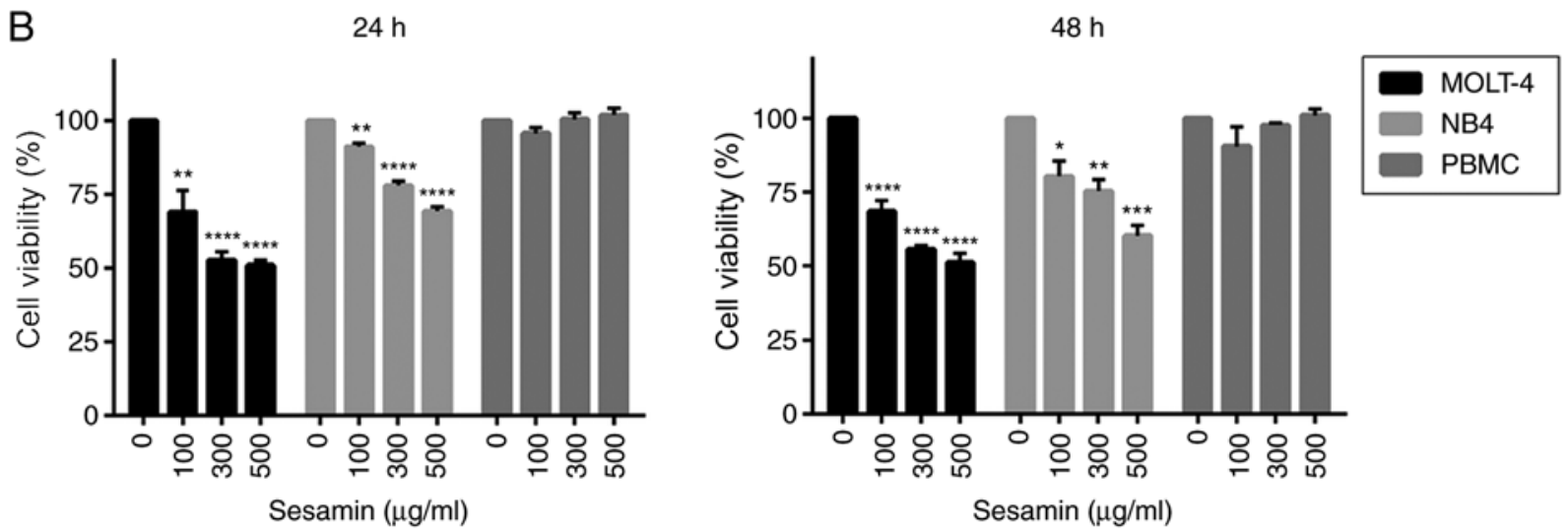

Figure 1. Sesamin suppresses the viability of leukemic cells. (A) Chemical structure of sesamin. (B) MOLT-4 and NB4 leukemic cells, and healthy PBMCs were treated with sesamin for 24 and 48 h. Cell viability was examined using an MTT assay. ${ }^{*} \mathrm{P}<0.05,{ }^{* * *} \mathrm{P}<0.01,{ }^{* * * *} \mathrm{P}<0.001$ and ${ }^{* * * * *} \mathrm{P}<0.0001$ vs. control $(0 \mu \mathrm{g} / \mathrm{ml})$. PBMC, peripheral blood mononuclear cell.

(GraphPad Software, Inc.). $\mathrm{P}<0.05$ was considered to indicate a statistically significant difference.

\section{Results}

Sesamin selectively exhibits cytotoxicity to leukemic cells. The chemical structure of sesamin is shown in Fig. 1A. To determine the anti-leukemic potential of sesamin, MOLT-4 and NB4 cells were treated with various concentrations of sesamin $(0,100,300$ and $500 \mu \mathrm{g} / \mathrm{ml})$. Following 24 and $48 \mathrm{~h}$ of incubation, cell viability was examined using an MTT assay. Sesamin significantly decreased the viability of MOLT-4 and NB4 cells in a dose-dependent manner (Fig. 1B). The $\mathrm{IC}_{50}$ value of sesamin at $48 \mathrm{~h}$ of incubation was 400 and $600 \mu \mathrm{g} / \mathrm{ml}$ in the MOLT-4 and NB4 cells, respectively. Notably, sesamin did not affect the viability of healthy PBMCs (Fig. 1B).

Sesamin induces the apoptosis of MOLT-4 and NB4 cells. To investigate the apoptosis induced by sesamin, MOLT-4 and NB4 leukemic cells were treated with sesamin at their $\mathrm{IC}_{50}$ concentration for $48 \mathrm{~h}$. The assessment of apoptosis was performed via Annexin V-FITC/PI staining and flow cytometry. The Annexin V-FITC ${ }^{+}$cells in Q2 (late apoptotic cells) and Q4 (early apoptotic cells) were defined as total apoptotic cells. The results revealed that sesamin significantly induced apoptosis of MOLT-4 and NB4 cells (Fig. 2A and B).

Sesamin induces autophagy by increasing the LC3-II level. To evaluate the induction of autophagy, leukemic cells were treated with sesamin at their $\mathrm{IC}_{50}$ concentration for $48 \mathrm{~h}$, and autophagy assay was performed using flow cytometry. The detection was based on the level of LC3-II in the cells that accordingly indicated the level of autophagy. Compared with the control, sesamin significantly increased the LC3-II level by $\sim 1.6$-fold in both cell lines (Fig. 3A and B).

Sesamin contributes to the alteration of $m R N A$ expression involved in apoptosis and autophagy. To examine the alteration of genes resulting from sesamin treatment, the expression levels of key genes associated with apoptosis, namely CASP3, and autophagy, namely mTOR and ULK1, were evaluated. The results of mRNA expression analysis in both cells demonstrated that sesamin induced apoptosis through the upregulation of CASP3 expression, and induced autophagy through the downregulation of mTOR and upregulation of ULK1 expression (Fig. 4).

Protein-chemical interaction of sesamin predicted by STITCH. STITCH was used to predict the association between sesamin and the proposed proteins (CASP3, mTOR and ULK1). The results from STITCH software analysis revealed that sesamin exhibited an association with CASP3 and mTOR via nitric oxide synthase 3. Moreover, ULK1, which is directly downstream of mTOR, exhibited high-score interactions with the target of rapamycin complex subunit LST8 (MLST8), regulatory-associated protein of mTOR (RPTOR) and proline-rich AKT1 substrate 1 (AKT1S1), as well as low-score interactions with ras homolog enriched in brain (RHEB), rapamycin-insensitive companion of mTOR (RICTOR), eukaryotic translation initiation factor 4E-binding protein 1 (EIF4EBP1) and eukaryotic translation initiation factor $4 \mathrm{E}$ (EIF4E). This interaction network indicated that CASP3, mTOR and ULK1 served a role 

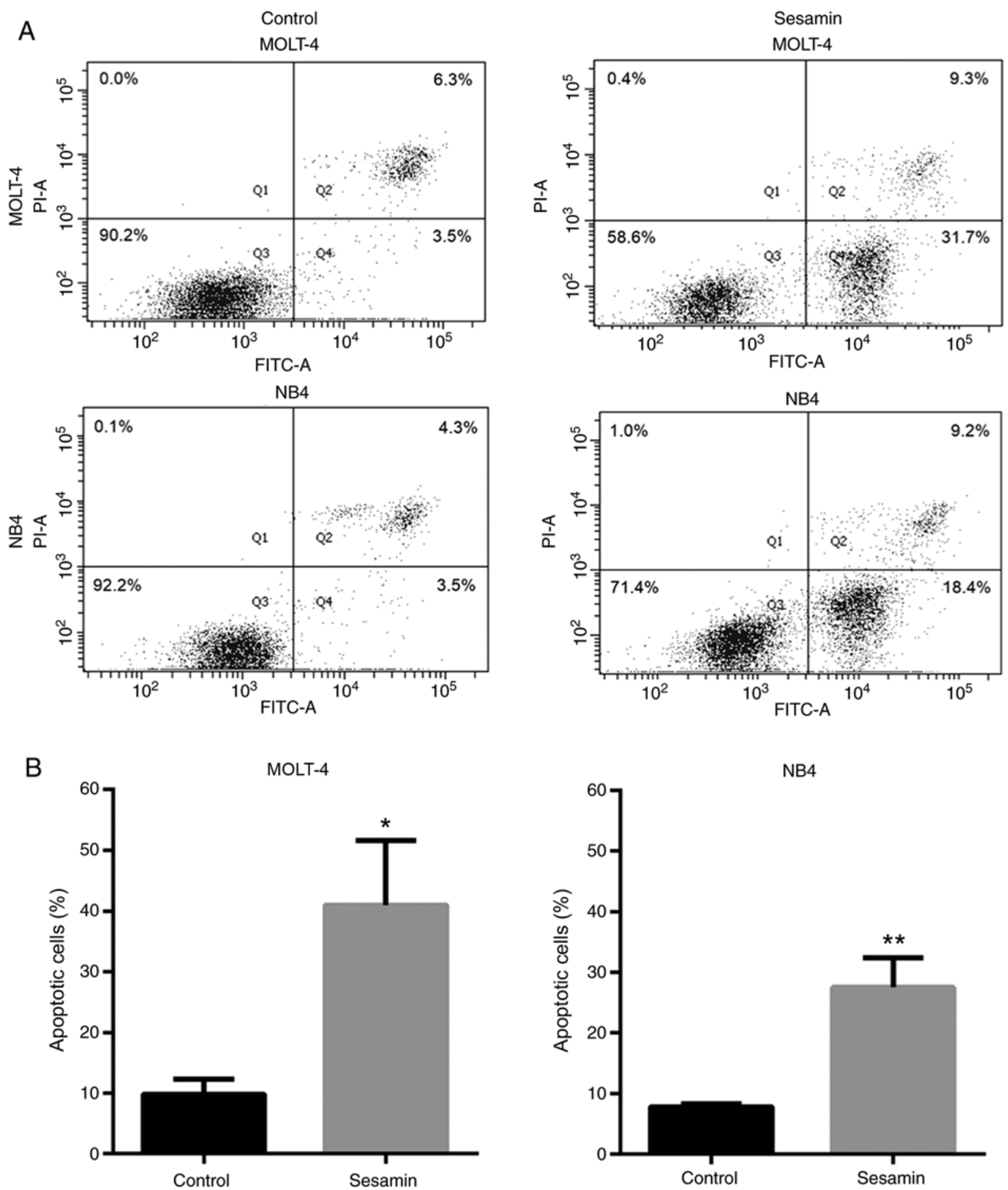

Figure 2. Sesamin induces the apoptosis of leukemic cells. (A) MOLT-4 and NB4 cells were incubated with sesamin at their IC 50 value for $48 \mathrm{~h}$, and apoptotic cells were analyzed via flow cytometry. Q1/upper left quadrant, necrotic cells; Q2/upper right quadrant, late apoptotic cells; Q3/lower left quadrant, viable cells; and Q4/lower right quadrant, early apoptotic cells. (B) Quantified results of apoptosis assay. " $\mathrm{P}<0.05$ and ${ }^{* * *} \mathrm{P}<0.01$ vs. control.

in apoptosis and autophagy in response to sesamin treatment, similar to rapamycin, AZD8055, Torin1 and Torin2 (Fig. 5).

\section{Discussion}

In the present study, the most substantial lignan in sesame seeds, known as sesamin, was investigated for its anti-leukemic effects on MOLT-4 and NB4 cell lines. It was revealed that sesamin exerted selective cytotoxic effects by inhibiting the viability of leukemic cells in a dose-dependent manner; however, it had a minimal effect on healthy PBMCs. Additionally, it increased apoptosis of both leukemic cell lines via the regulation of CASP3. When comparing the two cell lines, the MOLT-4 cells exhibited lower cell viability and a higher percentage of apoptosis compared with NB4 cells, suggesting that MOLT-4, an acute lymphoblastic leukemic cell line, was more sensitive to sesamin compared with NB4, an acute promyelocytic leukemic cell line. Previous studies 
A
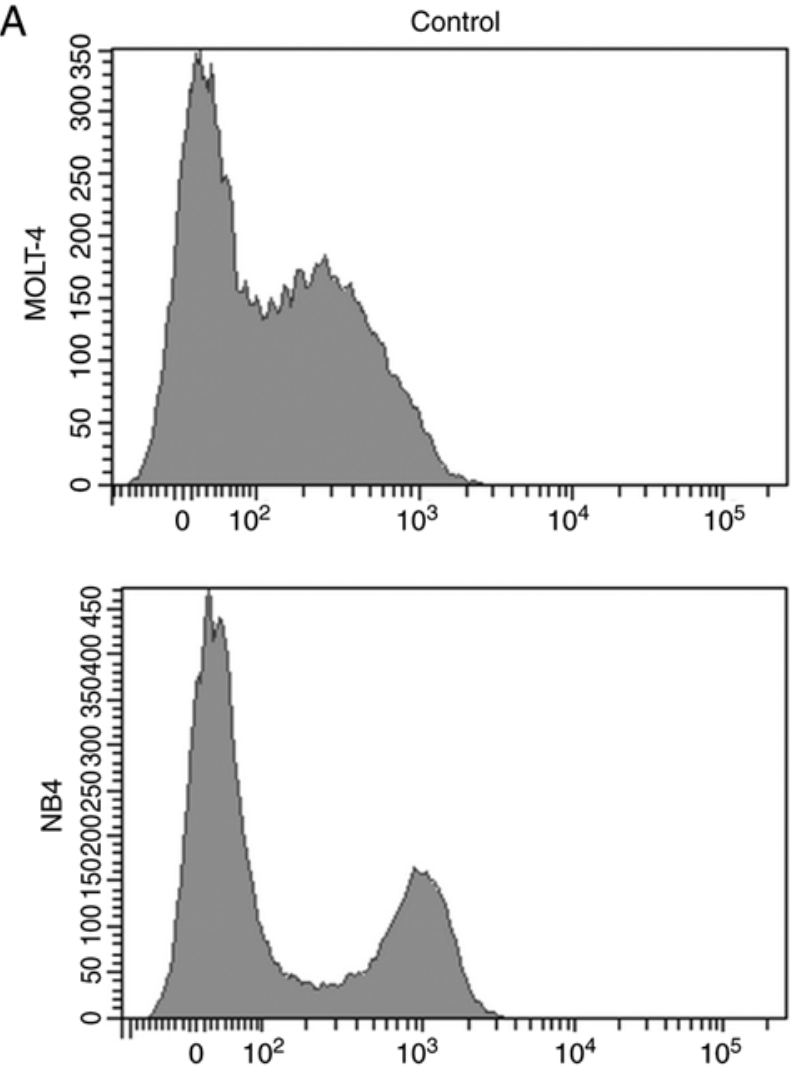
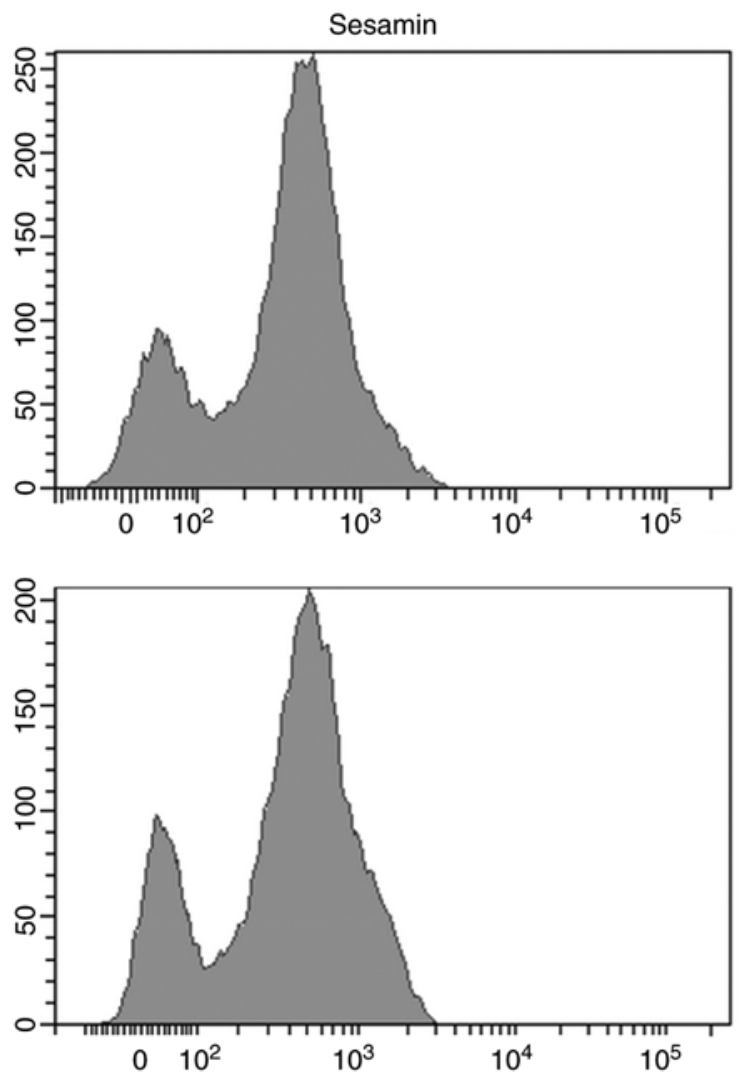

Fluorescence intensity

B

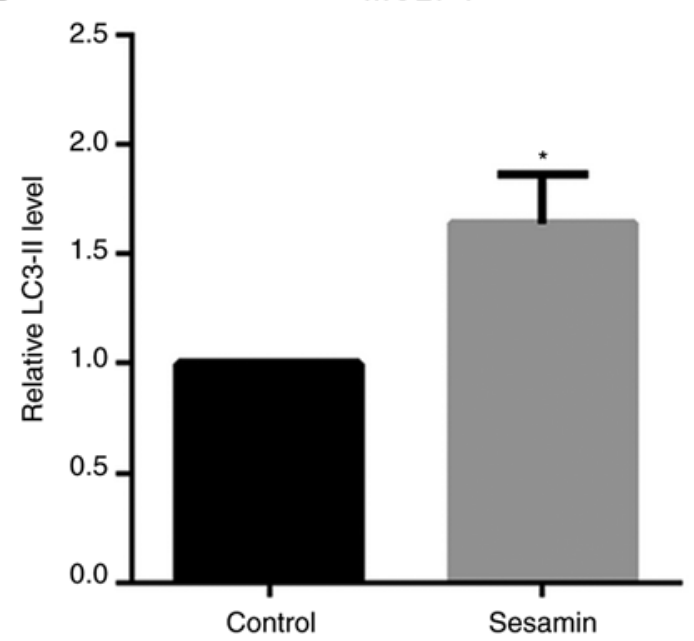

NB4

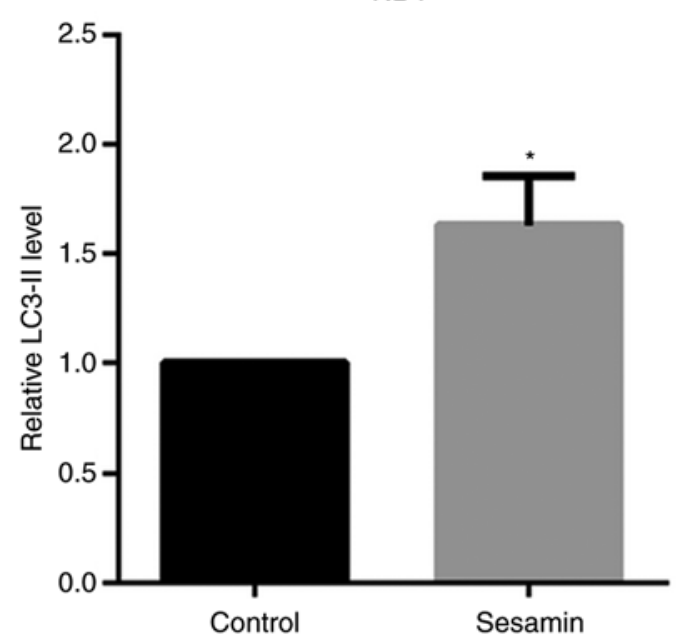

Figure 3. Sesamin induces autophagy in leukemic cells. (A) MOLT-4 and NB4 cells were treated with their IC ${ }_{50}$ concentration of sesamin for 48 h. Autophagy was quantitatively measured as the mean fluorescence intensity of LC3-II via flow cytometry. (B) Quantified results of autophagy assay. ${ }^{*} \mathrm{P}<0.05$ vs. control.

have reported the induction of apoptosis by sesamin in several types of cancer. For example, the regulation of the apoptotic proteins $\mathrm{Bax} / \mathrm{Bcl}-2$ and endoplasmic reticulum stress proteins in the inositol-requiring enzyme $1 \alpha / \mathrm{JNK}$ signaling pathway have been reported as key factors in cervical cancer cell apoptosis induced by sesamin (14). Additionally, sesamin is able to induce cell cycle arrest and apoptosis through the inhibition of STAT3 in hepatocellular carcinoma HepG2 cells (19).

In addition, previous studies have focused on the induction of autophagic cancer cell death by natural products. For example, ailanthone, the extract from Ailanthus altissima, induces autophagic cell death through the modulation of Beclin1, p62 and LC3 expression in HL-60 cells (20). Allicin and diallyl disulfide, the bioactive compounds in garlic extract, promote the mTOR-mediated autophagic death of human liver cancer and leukemic cells $(21,22)$. mTOR is a protein kinase that serves a role in cell proliferation, survival, metabolism and autophagy; the activation of mTOR is frequently observed in cancer (23). Thereby, the inhibition of mTOR has been considered for cancer therapy (24). ULK1 is an autophagic marker, and its downstream signals are triggered by decreased mTOR levels; it has been reported as a 
CASP3

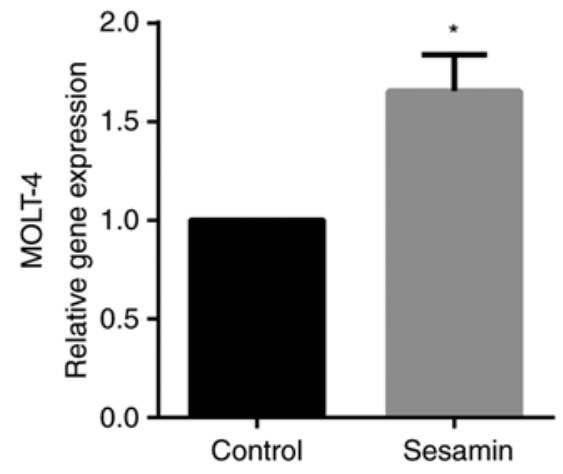

CASP3

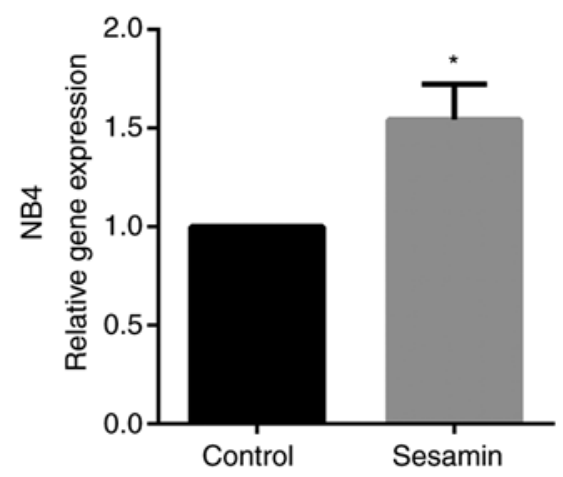

mTOR

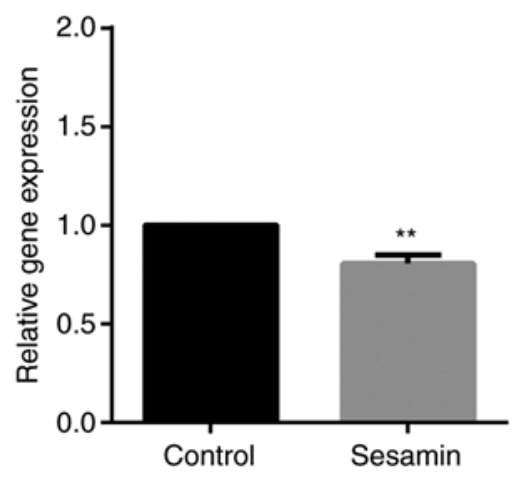

mTOR

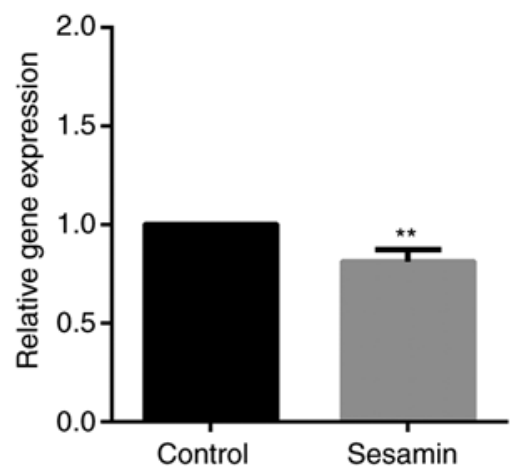

ULK1

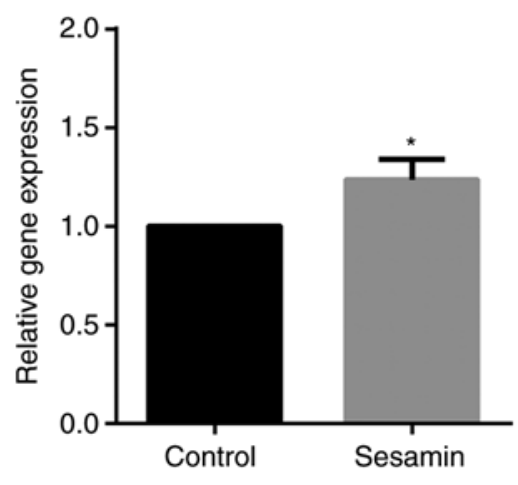

ULK1

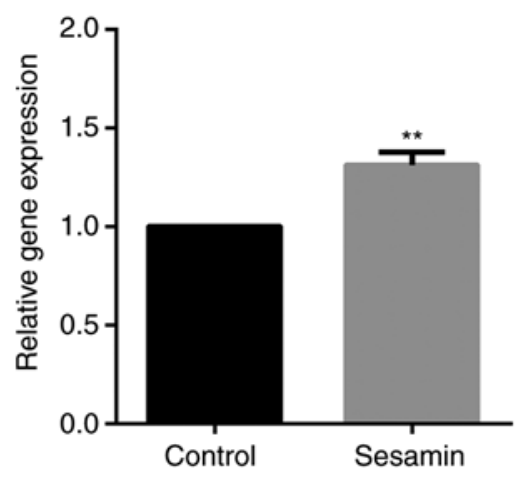

Figure 4. Sesamin induces leukemic cell death via the regulation of apoptotic and autophagic genes. Leukemic cells were incubated with sesamin at their $\mathrm{IC}_{50}$ value for $48 \mathrm{~h}$. The mRNA expression levels of CASP3, mTOR and ULK1 were examined via reverse transcription-quantitative PCR. ${ }^{*} \mathrm{P}<0.05$ and ${ }^{* * *} \mathrm{P}<0.01$ vs. control. CASP3, caspase 3; ULK1, unc-51 like autophagy activating kinase 1.

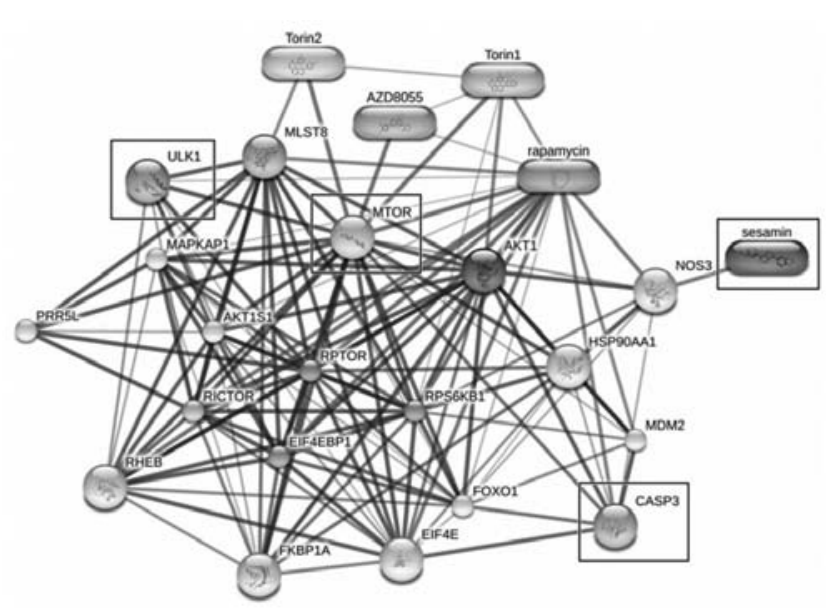

Figure 5. Prediction of protein-chemical interaction using the Search Tool for Interactions of Chemicals with the input of sesamin and associated factors (CASP3, mTOR and ULK1). Thick lines represent strong associations, while thin lines represent weak interactions. CASP3, caspase 3; ULK1, unc-51 like autophagy activating kinase 1; NOS3, nitric oxide synthase 3; MLST8, target of rapamycin complex subunit LST8; RPTOR, regulatory-associated protein of mTOR; AKT1S1, proline-rich AKT1 substrate 1; RHEB, ras homolog enriched in brain; RICTOR, rapamycin-insensitive companion of mTOR; EIF4EBP1, eukaryotic translation initiation factor 4E-binding protein 1; EIF4E, eukaryotic translation initiation factor 4E.

promising target for cancer therapy (25). Baicalein, a flavonoid from Scutellaria baicalensis, and LYN-1604, a candidate ULK1 agonist, exert antitumor effects through ULK1 activation in cancer cells $(26,27)$. The results of the present study demonstrated that sesamin treatment stimulated autophagy, represented by the increased levels of LC3-II in MOLT-4 and NB4 leukemic cells. This effect was accompanied by the downregulation of mTOR and the upregulation of ULK1 expression. This suggested that the induction of autophagy through mTOR/ULK1 signaling by sesamin may be an alternative treatment option for leukemia.

Bioinformatics tools have facilitated the comprehensive overview of available databases that are helpful to the understanding of pharmacology and biochemistry. The study of the interaction between proteins and small molecules is essential for the development of therapeutic drugs for diseases (28). The association between sesamin and possible associated molecules (CASP3, mTOR and ULK1) demonstrated that several targets participated in the response to sesamin. MLST8, RPTOR and AKT1S1, which exhibited strong interaction networks, were recognized as subunits of mTOR and their overexpression has been reported in a number of types of cancer cells, including colon, prostate, breast and lung cancer cells (29-31). It has been reported that the downregulation of MLST8, RPTOR and AKT1S1 expression inhibited tumor growth and induced cell death in colon cancer, lymphoma and sarcoma $(29,30,32,33)$. RHEB and RICTOR, which exhibited an interaction in the current network, are also involved in the mTOR signaling pathway (24). The deletion of RHEB and RICTOR can enhance cell cycle arrest and apoptosis of acute leukemic cells $(34,35)$. The other identified associated proteins, the translation factors EIF4E and EIF4EBP1, affect leukemogenesis by promoting growth and impairing the differentiation 
of blood cell precursors (36). Additionally, STITCH predicted that other anticancer agents, including rapamycin, AZD8055, Torin1 and Torin2, exhibited similar mechanisms to sesamin. These chemicals are categorized as mTOR inhibitors with anti-leukemic properties. Rapamycin treatment enhances the anti-neoplastic effects of the chemotherapeutic drug arabinozide cytarabine in acute myeloid leukemia (AML) (37). AZD8055 exerts cytotoxic effects in clinical AML cells through cell cycle blocking, caspase-dependent apoptosis and autophagic cell death (38). The mTOR inhibitors Torin1 and 2 have been investigated for their anticancer effects, revealing that they can suppress cell proliferation and promote the death of colon cancer and hepatocellular carcinoma cells $(39,40)$ and have thus progressed to clinical trials (41).

The present study suggested that sesamin may be a potent anti-leukemic agent, since it exerted anti-proliferative effects on MOLT-4 and NB4 leukemic cell lines. Further experiments indicated that these effects were mediated via the modulation of apoptosis and autophagy. The expression levels of the effective apoptotic factor CASP3 were increased in sesamin-treated cells. Autophagy occurred through the regulation of LC3-II triggered via the mTOR and ULK1 signaling pathway. The protein-chemical interaction analysis indicated that CASP3, mTOR and ULK1 were associated with several biomolecules in response to sesamin. The underlying mechanisms of sesamin were associated with the effects of the anti-neoplastic drug rapamycin and those of the well-known agents AZD8055, Torin 1 and 2.

The findings of the present study demonstrated the anti-leukemic effects of sesamin on MOLT-4 and NB4 cell lines through apoptotic and autophagic signaling pathways. These findings may be beneficial for the improved understanding of the role of sesamin in human leukemia. However, the present study only performed in vitro experiments. Thus, further studies on the effects of sesamin in vivo are required in the future for the development of alternative therapeutic strategies for leukemia.

\section{Acknowledgements}

Not applicable.

\section{Funding}

The present study was supported by the Royal Golden Jubilee PhD scholarship (grant no. PHD/0051/2558) from the Thailand Research Fund.

\section{Availability of data and materials}

The datasets used and/or analyzed during the current study are available from the corresponding author on reasonable request.

\section{Authors' contributions}

DT was involved in the conception of the study. KD and DT were involved in the experimental procedures. KD, CC, SR, UA and DT were involved in the interpretation and discussion of the results. KD was involved in the manuscript preparation. SR and DT were involved in the revision of the manuscript.
All authors have read and approved the final version of the manuscript.

\section{Ethics approval and consent to participate}

The experiments involving human blood samples were approved by the Committee for Research of the Faculty of Medicine Ramathibodi Hospital, Mahidol University (Bangkok, Thailand; approval no. MURA2019/679). All healthy adult volunteers provided written informed consent for donating peripheral blood.

\section{Patient consent for publication}

Not applicable.

\section{Competing interests}

The authors declare that they have no competing interests.

\section{References}

1. Wong SW, Lenzini S and Shin JW: Perspective: Biophysical regulation of cancerous and normal blood cell lineages in hematopoietic malignancies. APL Bioeng 2: 031802, 2018.

2. Bray F, Ferlay J, Soerjomataram I, Siegel RL, Torre LA and Jemal A: Global cancer statistics 2018: GLOBOCAN estimates of incidence and mortality worldwide for 36 cancers in 185 countries. CA Cancer J Clin 68: 394-424, 2018.

3. Ramirez LY, Huestis SE, Yap TY, Zyzanski S, Drotar D and Kodish E: Potential chemotherapy side effects: What do oncologists tell parents? Pediatr Blood Cancer 52: 497-502, 2009.

4. Saedi TA, Md Noor S, Ismail P and Othman F: The effects of herbs and fruits on leukaemia. Evid Based Complement Alternat Med 2014: 494136, 2014.

5. Moazzami AA, Haese SL and Kamal-Eldin A: Lignan contents in sesame seeds and products. Eur J Lipid Sci Technol 109: 1022-1027, 2007.

6. Ruankham W, Suwanjang W, Wongchitrat P, Prachayasittikul V, Prachayasittikul S and Phopin K: Sesamin and sesamol attenuate $\mathrm{H}_{2} \mathrm{O}_{2}$-induced oxidative stress on human neuronal cells via the SIRT1-SIRT3-FOXO3a signaling pathway. Nutr Neurosci: 1-12, 2019 (Epub ahead of print).

7. Kong P, Chen G, Jiang A, Wang Y, Song C, Zhuang J, Xi C, Wang G, Ji Y and Yan J: Sesamin inhibits IL-1 $\beta$-stimulated inflammatory response in human osteoarthritis chondrocytes by activating Nrf2 signaling pathway. Oncotarget 7: 83720-83726, 2016.

8. Miyawaki T, Aono H, Toyoda-Ono Y, Maeda H, Kiso Y and Moriyama K: Antihypertensive effects of sesamin in humans. J Nutr Sci Vitaminol (Tokyo) 55: 87-91, 2009.

9. Liang YT, Chen J, Jiao R, Peng C, Zuo Y, Lei L, Liu Y, Wang X, Ma KY, Huang Y and Chen ZY: Cholesterol-lowering activity of sesamin is associated with down-regulation on genes of sterol transporters involved in cholesterol absorption. J Agric Food Chem 63: 2963-2969, 2015.

10. Majdalawieh AF, Massri M and Nasrallah GK: A comprehensive review on the anti-cancer properties and mechanisms of action of sesamin, a lignan in sesame seeds (Sesamum indicum). Eur J Pharmacol 815: 512-521, 2017.

11. Fang Q, Zhu Y, Wang Q, Song M, Gao G and Zhou Z: Suppression of cyclooxygenase 2 increases chemosensitivity to sesamin through the Akt-PI3K signaling pathway in lung cancer cells. Int J Mol Med 43: 507-516, 2019.

12. Siao AC, Hou CW, Kao YH and Jeng KC: Effect of sesamin on apoptosis and cell cycle arrest in human breast cancer mcf-7 cells. Asian Pac J Cancer Prev 16: 3779-3783, 2015.

13. Tanabe H, Kuribayashi K, Tsuji N, Tanaka M, Kobayashi D and Watanabe N: Sesamin induces autophagy in colon cancer cells by reducing tyrosine phosphorylation of EphA1 and EphB2. Int J Oncol 39: 33-40, 2011. 
14. Dou H, Yang S, Hu Y, Xu D, Liu L and Li X: Sesamin induces ER stress-mediated apoptosis and activates autophagy in cervical cancer cells. Life Sci 200: 87-93, 2018.

15. Banjerdpongchai R, Yingyurn S and Kongtawelert P: Sesamin induces human leukemic cell apoptosis via mitochondrial and endoplasmic reticulum stress pathways. World J Oncol 1: 78-86, 2010.

16. Harikumar KB, Sung B, Tharakan ST, Pandey MK, Joy B, Guha S, Krishnan S and Aggarwal BB: Sesamin manifests chemopreventive effects through the suppression of NF-kappa B-regulated cell survival, proliferation, invasion, and angiogenic gene products. Mol Cancer Res 8: 751-761, 2010.

17. Livak KJ and Schmittgen TD: Analysis of relative gene expression data using real-time quantitative PCR and the 2(-Delta Delta C(T)) method. Methods 25: 402-408, 2001

18. Kuhn M, von Mering C, Campillos M, Jensen LJ and Bork P: STITCH: Interaction networks of chemicals and proteins Nucleic Acids Res 36: D684-D688, 2008.

19. Deng P, Wang C, Chen L, Wang C, Du Y, Yan X, Chen M, Yang $\mathrm{G}$ and He G: Sesamin induces cell cycle arrest and apoptosis through the inhibition of signal transducer and activator of transcription 3 signalling in human hepatocellular carcinoma cell line HepG2. Biol Pharm Bull 36: 1540-1548, 2013.

20. Wei C, Chen C, Cheng Y, Zhu L, Wang Y, Luo C, He Y, Yang Z and Ji Z: Ailanthone induces autophagic and apoptotic cell death in human promyelocytic leukemia HL-60 cells. Oncol Lett 16: 3569-3576, 2018

21. Chu YL, Ho CT, Chung JG, Rajasekaran R and Sheen LY: Allicin induces p53-mediated autophagy in Hep G2 human liver cancer cells. J Agric Food Chem 60: 8363-8371, 2012.

22. Suangtamai $T$ and Tanyong DI: Diallyl disulfide induces apoptosis and autophagy via mTOR pathway in myeloid leukemic cell line. Tumour Biol 37: 10993-10999, 2016.

23. Kim J and Guan KL: mTOR as a central hub of nutrient signalling and cell growth. Nat Cell Biol 21: 63-71, 2019.

24. Hua H, Kong Q, Zhang H, Wang J, Luo T and Jiang Y: Targeting mTOR for cancer therapy. J Hematol Oncol 12: 71, 2019.

25. Liu L, Yan L, Liao N, Wu WQ and Shi JL: A review of ULK1-mediated autophagy in drug resistance of cancer. Cancers (Basel) 12: 352, 2020.

26. Aryal P, Kim K, Park PH, Ham S, Cho J and Song K: Baicalein induces autophagic cell death through AMPK/ULK1 activation and downregulation of mTORC1 complex components in human cancer cells. FEBS J 281: 4644-4658, 2014.

27. Zhang L, Fu L, Zhang S, Zhang J, Zhao Y, Zheng Y, He G, Yang S, Ouyang L and Liu B: Discovery of a small molecule targeting ULK1-modulated cell death of triple negative breast cancer in vitro and in vivo. Chem Sci 8: 2687-2701, 2017.

28. Xia X: Bioinformatics and drug discovery. Curr Top Med Chem 17: 1709-1726, 2017.

29. Kakumoto K, Ikeda J, Okada M, Morii E and Oneyama C: mLST8 promotes mTOR-mediated tumor progression. PLoS One 10: e0119015, 2015.
30. Gulhati P, Cai Q, Li J, Liu J, Rychahou PG, Qiu S, Lee EY, Silva SR, Bowen KA, Gao T and Evers BM: Targeted inhibition of mammalian target of rapamycin signaling inhibits tumorigenesis of colorectal cancer. Clin Cancer Res 15: 7207-7216, 2009.

31. Malla R, Ashby CR Jr, Narayanan NK, Narayanan B, Faridi JS and Tiwari AK: Proline-rich AKT substrate of 40-kDa (PRAS40) in the pathophysiology of cancer. Biochem Biophys Res Commun 463: 161-166, 2015.

32. Martin R, Desponds C, Eren RO, Quadroni M, Thome M and Fasel N: Caspase-mediated cleavage of raptor participates in the inactivation of mTORC1 during cell death. Cell Death Discov 2: $16024,2016$.

33. Lv D, Liu J, Guo L, Wu D, Matsumoto K and Huang L: PRAS40 deregulates apoptosis in Ewing sarcoma family tumors by enhancing the insulin receptor/Akt and mTOR signaling pathways. Am J Cancer Res 6: 486-497, 2016.

34. Gao Y, Gao J, Li M, Zheng Y, Wang Y, Zhang H, Wang W, Chu Y, Wang X, Xu M, et al: Rhebl promotes tumor progression through mTORC1 in MLL-AF9-initiated murine acute myeloid leukemia. J Hematol Oncol 9: 36, 2016.

35. Hua C, Guo H, Bu J, Zhou M, Cheng H, He F, Wang J, Wang X, Zhang Y, Wang Q, et al: Rictor/mammalian target of rapamycin 2 regulates the development of Notch1 induced murine T-cell acute lymphoblastic leukemia via forkhead box O3. Exp Hematol 42: 1031-1040.e1-e4, 2014.

36. Topisirovic I, Guzman ML, McConnell MJ, Licht JD, Culjkovic B, Neering SJ, Jordan CT and Borden KLB: Aberrant eukaryotic translation initiation factor 4E-dependent mRNA transport impedes hematopoietic differentiation and contributes to leukemogenesis. Mol Cell Biol 23: 8992-9002, 2003.

37. Janus A, Linke A, Cebula B, Robak T and Smolewski P: Rapamycin, the mTOR kinase inhibitor, sensitizes acute myeloid leukemia cells, HL-60 cells, to the cytotoxic effect of arabinozide cytarabine. Anticancer Drugs 20: 693-701, 2009.

38. Willems L, Chapuis N, Puissant A, Maciel TT, Green AS, Jacque N, Vignon C, Park S, Guichard S, Herault O, et al: The dual mTORC1 and mTORC2 inhibitor AZD8055 has anti-tumor activity in acute myeloid leukemia. Leukemia 26: 1195-1202, 2012.

39. Francipane MG and Lagasse E: Selective targeting of human colon cancer stem-like cells by the mTOR inhibitor Torin-1. Oncotarget 4: 1948-1962, 2013.

40. Wang C, Wang X, Su Z, Fei H, Liu X and Pan Q: The novel mTOR inhibitor Torin-2 induces autophagy and downregulates the expression of UHRF1 to suppress hepatocarcinoma cell growth. Oncol Rep 34: 1708-1716, 2015.

41. Sun SY: mTOR kinase inhibitors as potential cancer therapeutic drugs. Cancer Lett 340: 1-8, 2013.

This work is licensed under a Creative Commons Attribution-NonCommercial-NoDerivatives 4.0 International (CC BY-NC-ND 4.0) License. 